\title{
Looking at Language, Identity, and Mobility in Suriname
}

Eithne B. Carlin, Isabelle Léglise, Bettina Migge and Paul B. Tjon Sie Fat

\section{$1 \quad$ Introduction}

Language in Suriname is a vigorous obsession and has been an emotive topic since colonisation by the Dutch. Today, Dutch continues to be the sole officially recognised and promoted language while the vast majority of the population speaks any number of the other 20 or more languages found in Suriname, though not necessarily including Dutch. Popular and official discourse on language, in the main, revolve around language ideologies that are steeped in the colonised mindset of ethnicised inequality whereby the importance of knowing Dutch is regarded as having gate-keeping functions in Surinamese society. The other languages tend to be associated with ethnic and social constructs that are not conducive to upward social mobility, but many of them are indispensible for managing everyday life and tend to have high covert prestige.

Previous scholarship on some of the individual languages of Suriname and on language in Suriname, has, in the main, focused on historical issues such as language genesis (see, for example, Arends 1995; Migge 2003; articles in Carlin and Arends 2002; Migge and Smith 2007 and in Essegbey, Migge and Winford 2013 for works on the creole languages of Suriname), the historical development of, in particular, Sranantongo (Arends 1989; Bruyn 1995; van den Berg 2007) and language description (Carlin 2004; Huttar and Huttar 1994; articles in Carlin and Arends 2002; Goury and Migge 2003; McWhorter and Good 2012). Earlier work presented in Charry et al. (1983) provides some useful information about how Dutch, Sranantongo and Sarnámi were practiced, including multilingual practices and contact patterns, language ideologies and their recent development. There are also a few articles that examine the linguistic context of Suriname based on statistical (census) and socio-historical data by St-Hilaire $(1999,2001)$ who has argued that Dutch is gaining ground in Suriname due to a policy of linguistic assimilation. Assimilation, however, for as far as it is taking place, has not proceeded at the same speed and in the same way for all Surinamese. Crucially, urbanised populations tend to have 
a greater knowledge of Dutch and consequently contact between Dutch and the languages spoken by urbanised populations, as well as mutual contact between the latter is much more intense in the main urban hubs, and Paramaribo in particular, than in rural locations. However, linguistic diversity and contact, as we show in this book, are not solely characteristics of urban spaces. Outside of Paramaribo, the use of languages other than Dutch tends to be more the norm. While these previous works are clearly valuable, there is a need to update them with current data and to expand the focus of attention beyond the urban centres and mainstream cultural and linguistic contact situations to those languages and populations that are often considered to be peripheral in the Surinamese imagination, namely the languages spoken by rural populations and more recent immigrants.

This book aims, therefore, at revisiting the social and linguistic context of contemporary Suriname and shifting attention away from the purely historical and anthropological construction of Surinamese reality to look instead at language practices in Suriname through the lens of identity construction, mobility patterns, linguistic ideology and multilingualism. The three main themes we engage in this book, language, identity and mobility overlap in several aspects, though the link between language and social identity would likely seem the most obvious for most people. From an evolutionary point of view, the huge variety of living languages and varieties of the same language can be related to the human need to index group identity; language helps to bind us to those with whom we share primal group identity, and it separates us from outsiders and competing groups (Pagel 2012). In dominant Surinamese multiculturalist discourse, ethnicity and language are interchangeable; ethnic identity implies a distinct language used by a particular ethnic group, and languages are often thought to reflect monolithic ethnic identities. In practice, this is, of course, not the case because people who claim certain ethnic belonging, for example, Hindustani, do not necessarily also claim to speak Sarnámi as their main language or even at all.

Surinamese are generally multilingual. They creatively draw on a range of languages and language practices in order to (temporarily) invoke certain identities, stances, and relationships and to (re)negotiate existing social constructs. The types of languages that are practiced and their social functions are variable across individuals depending both on people's aspirations and the social networks and contexts in which they interact. In the dominant Surinamese discourse of language and identity, which is reflected in state and non-state institutions, the media and education, and entrenched in historical ideologies and economic practices, mobility has come to be, paradoxically enough, a static notion, one that refers to the historical labour importation into 
Suriname, first from Africa and later from Asia, and migration from Suriname towards the Netherlands and elsewhere. Neither modern globalised migration nor fundamental motivations of human mobility, such as curiosity, wanderlust, and the like, that fall outside of the historical construct of the nation state, are recognised in Suriname as being mobility. For example, recent Chinese migration to Suriname is seen as a continuation of a uniquely Surinamese process that began in the mid-nineteenth century with the advent of Chinese indentured labourers installed to replace slave labour after the abolition of slavery, rather than as the result of the worldwide impact of Chinese globalisation. It is in this light that mobility patterns of, for example, Amerindians in the interior of Suriname and surrounding countries, or movements of Maroons along and across the Marowijne border are barely recognised as being mobility at all; rather in the former case they are seen as essentialist features of an imagined nomadic identity, and in the latter case they do not figure in an equally imaginary sedentary, tribal identity. The Maroon and Amerindian mobility patterns fall outside of the historicised peopling of mainstream, or urban, Suriname. However, these movements from village to village, from kampu to kampu, have always been basic to, and constitutive of, the historical peopling of the Guianas.

Methodologically, social science research in Suriname has been limited by the idiosyncrasies of the ethnicised view of the Surinamese state, where the nation is taken as the prime container category, an arbitrarily bounded context. In order to avoid methodological territorialism, namely "formulating concepts and questions, constructing hypotheses, gathering and interpreting empirical evidence, and drawing conclusions all in a territorial spatial framework" (Scholte 2005), this book takes social interactions and social actors as primary categories. Traditionally, post-modernist and post-structuralist approaches to Surinamese society are rare (for one example, see Tjon Sie Fat 2009a), and the use of ethnic groups as valid analytical categories in social science and linguistic research is seldom challenged. This book breaks away from the traditional notions of bounded ethnic groups and the tug of the urban centres to show interwoven social interactions that are constitutive of identity-making processes and ever-changing linguistic practices.

\section{Identity Construction}

Identity can be broadly defined as a person's sense of belonging to or alignment with a specific social group, society or place, and identity construction as the ways in which people negotiate this belonging or alignment. Identities are generally variable, contingent, and emergent rather than immutable. People 
claim membership in multiple groupings encompassing "(a) macro-level demographic categories; (b) local, ethnographically specific cultural positions; and (c) temporary and interactionally specific stances and participant roles" (Bucholtz and Hall 2010: 21). Dealing with the issue of identity is very fundamentally a methodological concern. The cultural studies approach to cultural identity, which includes, but is not limited to, ethnic identity, is very much anti-essentialist; subjects are not unified but fractured, made up of multiple, changeable, contingent, situational identities rather than a single, fixed one (Hall 1990). People are not their social identities; rather, they perform or enact social identities (Butler 1990). And in social interactions identities are constantly being (re)negotiated. The different identities are linked to, but are not determined, in an essentialist manner, by the various social roles that people engage in across the different contexts in which they generally interact/participate. Thus, an Amerindian activist can promote Amerindian identity politics in his own language in his community and in Dutch in Paramaribo while at the same time ensuring the upward social mobility of his children by insisting on their being educated in Dutch, and maintaining his inter-ethnic networking skills by the use of Sranantongo. By using the different languages in his repertoire he can easily shift between identities on the local, regional, and national levels in the various and often simultaneously occurring contexts.

Language is generally assumed to be one of the most salient markers of identity, as it links people to places, communities, and ways of being in the world. Its constitution lies in indexicality which "involves the creation of semiotic links between linguistic [or non-linguistic] forms and social meanings (Ochs 1992; Silverstein 1985)". Commonly attested indexical processes involve: "(a) overt mention of identity categories and labels; (b) implicatures and presuppositions regarding one's own or other's identity position; (c) displayed evaluative and epistemic orientations to ongoing talk, as well as interactional footings and participant roles; and (d) the use of linguistic structures and systems that are ideologically associated with specific personas and groups" (Bucholtz and Hall 2010: 21). In other words, specific languages, ways of using a language, ways of talking about languages and/or linguistic properties become indices of social groups or identities through the processes of iconisation and erasure (Irvine and Gal 2000: 37). Social identities cannot be described independently of their temporal, social and spatial context. Thus, the way in which group boundaries are marked and negotiated through linguistic practices (cf. Barth 1969; Wimmer 2013), needs to be explored through both micro- and macro-level empirical social and linguistic research that focuses on people's actions and people's understandings or perceptions of their own and other's actions. As shown in Léglise and Migge (2006) and Migge and Léglise (2013), 
examining the perspectives of all social actors allows us to elucidate the complex links between social and linguistic behaviour.

With regard to ethnic identity, such a tendency to treat ethnic groups as emic rather than etic categories is called 'groupism' (Brubaker 2004). Ethnic groups are not bounded entities, rather they are social constructs that are invoked for specific purposes in specific contexts. Ethnic identities, just like any other identities, are therefore highly fluid, relational, and situational. For example, Maroons may identify with particular clans and ethnic groups in the interior of Suriname but may assume a more general Maroon identity in Paramaribo, where they easily access an even wider Afro-Surinamese identity. There is an absence of studies of Surinamese society that take as their focus identity as a fluid social construct, be that ethnic, gender, class or any other identity. Assimilation, hybridity, and ethnic intertwining, for example through mixed unions, are not reflected sufficiently in current scholarship on Suriname, particularly historical and ethnographic works, which still too often reflect the dominant ethnicised discourse which assumes ethnic identity to be a bounded measurable entity. These are not, however, just a fact of the recent past although it would seem that processes of urbanisation have led to an increase in intensity of 'ethnic' and social mixing. All the authors in this book have engaged with the challenge of avoiding methodological territorialism as described above, and have taken pains to highlight the tenuous link that exists between ethnicity and other social categories and constructs such as language.

Tjon Sie Fat, for example, discusses the mismatch between the idea of a monolithic Chinese ethnicity with an associated, and equally monolithic Chinese language, and the reality of increased linguistic variety as a result of immigration from many different areas in China since the early 199os. A single label, 'Chinese', covers different regional backgrounds and dialects spoken by New Chinese migrants in Suriname. A similar mismatch between a popular label, Amerindian, and a complex reality is the background to Carlin and Mans' discussion of the multiplicity of identities hidden underneath Amerindian ethnonyms in southern Suriname. They show that identities in the various ethnic hubs are more than simple lists of available labels. Rather, all relevant identities exist in a Matryoshka doll fashion, and previously dormant identities may become reactivated when context and locality change.

The mismatch between ethnonyms and language labels is also raised by Léglise and Migge in their discussion of language ideology among Surinamese schoolchildren. Their unique study shows how widespread and regionally variable multilingualism goes hand in hand with a variety of (situationally different) language names to make any straightforward pairing of language and ethnic identity untenable. 
Van den Berg, Borges and Yakpo also give lie to the simplistic notion of language as identity in the context of Suriname. They challenge the notion that Surinamese languages reflect fixed pluriformity by offering indications that some, if not many, of these languages are changing and influencing each other structurally thereby making the use of several languages in the same context easier as greater structural similarity increases interchangeability.

Yamada reports on a local scheme to revitalise a low-prestige variety of Kari'na, itself of low prestige in Surinamese society, in order to strengthen Indigenous identity within established multiculturalist discourse in Suriname. Her case study focusses on Konomerume (Donderkamp), a village that is consistently identified as Kari'na but which also in fact has a sizeable population of migrants from Suriname and abroad, and a concomitant linguistic complexity.

In many of the contributions in this book, there is a definite suggestion of language as a marker of class identity. Dutch as a prestige language associated with whiteness, the Netherlands, education, upper class, contrasts with Sranantongo, which is associated with low prestige, blackness, lack of economic success, and also with migrant and minority languages which indicate marginality. However, there is no straightforward relationship between language and social identity. Close observation of people's linguistic practices reveals a much more complex picture of identities and identity construction as people regularly claim different languages in the same, and across different contexts, and make use of one and the same language to negotiate different social identities (see the section on language ideology below).

The primary, literal meaning of mobility is human population movements. Human physical mobility may be defined as: "all forms of territorial movement by people. These movements take place at different spatial and temporal scales and reflect a wide range of underlying factors and motivations" (Alexiades 2009:2). These movements may be individual or of groups such as households, ethnic groups, even nations, though the continuum of collective mobility, between the extremes of nomadism and sedentism, is what we generally refer to as mobility. Mobility may also be voluntary or involuntary, temporary or permanent, cyclical or unidirectional, and different forms may occur simultaneously. Migration is mobility in a more restricted sense, implying movement from fixed communities to fixed destinations, institutionalisation via migrant organisations, against the backdrop of a nation-state, and the implicit 
notion that immobility is the neutral human condition. Transnationalism, by contrast, comes from the realisation that migration is not quite that simple, and that migrant networks and social fields transcend national boundaries under continuous mobility.

Five types of geographic mobility that overlap in part are relevant to a discussion of current linguistic developments in Suriname: mobility in the interior, urbanisation, transnational ties to the colonial metropolis, regional migration, new migration under globalisation.

Mobility may also be used in a metaphoric, non-geographic sense as social (upward and downward) mobility, and cultural mobility. In its more abstract sense, mobility refers to changes in human populations, such as movement between economic sectors, income levels, and social classes. In this sense, territorial movement becomes a subject of economic inquiry-how social mobility and economic change are linked to spatial movement and interaction between populations. Cultural mobility may also be linked to physical mobility; one can think of processes of acculturation, integration, and generational processes of assimilation that attend the reality of migration. These different forms of mobility are interrelated with issues of identity, and therefore language. For example, Léglise and Troiani (2011) show how Brazilians first travelled back and forth within Brazil, then between Brazil and French Guiana before finally settling there, and how this process is linked to opportunities, economic, social, and linguistic changes.

As is the case with language and identity, the study of human mobility is a study of changeable processes and complex networks. Social mobility can no longer be simply defined in terms of upward or downward mobility, nor can spatial mobility be reduced to immigration or emigration, nor should one conceive of identity and language solely in terms of migrant communities versus non-mobile settled groups. As one aspect of globalisation, modern migration (internal, regional, international, and anything in between) requires conceptual frameworks such as transnationalism theory that take into account that human mobility is neither unidirectional nor unbounded, just like the fluid social networks that enable it. Transnational social spaces should be the focus of analysis, not fixed categories of social groups or geographical locations (Faist 2000, Vertovec 1999). In this book we therefore also zoom in on social, cultural and linguistic contact patterns not only in the urban Surinamese setting but also between the 'other' players in Suriname, and reflect on how social categories other than ethnicity affect language practices.

The link between language and mobility is most evident in four chapters. Carlin and Mans remind us how historical mobility of the peoples who 
currently live in southern Suriname had — and has — very little to do with the notion of the Surinamese state and its national borders. They show how mobility shaped group identities through the fusion and fission of earlier groups. Collomb and Lescure combine anthropology and linguistics to paint a picture of this development for the Kari'na people of the coastal Guianas. The historical impact of migration and trade on the Kari'na has resulted in lexical borrowings from Spanish, Dutch, Sranantongo and French, and to a lesser extent in emergent syntactic innovations.

Laëthier and Tjon Sie Fat reflect on modern migration and its impact on the ethnic landscape. Laëthier reports on regional migration networks in the French Caribbean, namely the Haitian undertaking to reach French Guiana. She touches on Afro-Caribbean ethnic variation, and the introduction of French Caribbean language and culture in Suriname and the role of Surinamese creole languages in French Guiana. Tjon Sie Fat raises the issue of broader South-South migration in his discussion of the impact of New Chinese migrants in Suriname. Parallel to the rising regional influence of the People's Republic of China as a superpower, is the emergence of the standard language of the People's Republic, Putonghua, as the Chinese intra-ethnic lingua franca in Suriname.

Here we see transnationalism by default, the incorporation of Surinamese Chinese into 'Global Chineseness', the globalised Chinese cultural identity fostered by the Chinese State through the medium of Putonghua. Whereas Tjon Sie Fat only touches on the transnational circuits of New Chinese migration, Laëthier relies on the transnational social fields that shape Haitian patterns of mobility, settlement, and identity. Haitian identity discourse is structured around the basic flow from Haiti to French Guiana, and Suriname is considered a transit stop on that route. De Theije describes the Surinamese case of a wider phenomenon of Brazilian garimpeiros, artisanal gold miners, who represent a fairly straightforward instance of transnational flows of people, money, and culture, linking transnational social spaces in Suriname and northern Brazil.

Van Stipriaan also describes developments in the Surinamese interior, and increasing flows of people, money and ideas within Suriname. He describes the historical development of the growing contact between Maroon homelands and Paramaribo. Globalisation is increasingly impacting the interior, where changing transport and communications technologies are symbolic of rapid social transformation. He notes that Maroon identities and social structure are shifting under the influence of Paramaribo. While Van Stipriaan's axis of mobility is mainly South-North, de Theije describes East-West mobility across the Marowijne River between Suriname and French Guiana. Various ethnic groups (indigenous, local, and foreign) are constructing and reconstructing 
ethnic and class identities in the context of economic opportunities and livelihoods provided by the river.

Laëthier's Haitian case is also about crossing the Marowijne River, though the movement is predominantly eastward, to French Guiana. In their Surinamese sojourn and subsequent settlement in French Guiana, Haitian migrants find themselves reinterpreting Haitian identity in their encounters with various other Afro-Caribbean groups, in terms of race, ethnicity, and class. In contrast, Yamada describes immigration from the Surinamese coastal regions and Guyana to the relatively isolated village of Konomerume/Donderkamp and shows how it has resulted in a strongly hybrid community.

In this book the complex interplay between mobility, identity and language produces subtle and at times less subtle challenges to the idea of the nationstate and its national borders. This is most clearly apparent in de Theije's case of the Marowijne River, the official border between the Surinamese state and French Guiana, in fact the EU. Its role as the border between economically, socially, and institutionally mismatched polities creates opportunities in the informal economy and migration, and depending on the context and the actors involved, this may be constructed as smuggling and illegal migration. In Laëthier's contribution, migration via Suriname is an adaptation to institutional changes that affected earlier migrant networks and trajectories, and here too, as she shows, transit creates opportunities.

The challenges to the Surinamese state are also apparent in the cases involving minorities. Van Stipriaan shows how Maroon societies are being incorporated at an increasing pace by Surinamese society and also the state. Collomb and Lescure present the current division between Tyrewuju (eastern Kari'na) and Aretyry (western Kari'na) in terms of different social regimes, cultural policies, and institutions in the frontier regions on either side of the Marowijne River. Carlin and Mans essentially argue that Amerindian autonyms of southern Suriname are related to group identities that emerged far away from the Surinamese state in time and space, and make little sense in modern Surinamese national discourses that pose a monolithic 'Indigenous' category. Similarly, Tjon Sie Fat argues that the Surinamese state reproduces popular notions of ethnicity to recognise only one Chinese ethnic and therefore linguistic category.

\section{$4 \quad$ Multilingualism, Ideology and Language as Boundary}

Identity may emerge, for instance, from the construction of social borders and language may be one of many cultural boundary markers that populations 
use to show what they are and what they are not (Barth, 1969). Such borders are not rationally and democratically agreed, and unequal power relations determine the way different groups will be included or excluded (Eriksen 1993). Identity does not require actual groups to exist, rather all group identities are primarily the result of human agents 'doing' identity. Potential social cleavages may become politically salient, and cultural dispositions such as a mother tongue may become ethnicised-in fact, ethnicity constantly arises and changes in the everyday interaction between individuals, and actors have different options available to react to existing social boundaries. As Wimmer states, people act in order: "[ ... ] to overcome or reinforce them [social boundaries], to shift them, to exclude new groups of individuals or include others, or to promote other, nonethnic modes of classification and social practice" (Wimmer 2013: 46).

Language is also symbolic capital, and the ability to handle more languagesmultilingualism - will increase one's ability to compete for resources, regardless of whether or not those languages are pegged to one's 'core identity' and irrespective of their status (see Bourdieu 1982; 1991). Suriname, like many other countries, does not officially promote multilingualism (Migge and Léglise, in press). Elite multilingualism is promoted for global languages such as English or Spanish associated with a high potential economic value. In contrast, the economic value of local languages is not officially recognised. Languages such as Portuguese and Chinese, even if they are not seen as prestige languages, do have a certain economic value in some contexts and localities linked, for example, to small scale gold mining in the interior (see de Theije and Heemskerk 2009 and de Theije, this volume) or the Chinese retail trade.

Mobility, particularly in the form of migration, results in pluricultural competence, which virtually always entails multilingual competence. According to Coste et al. (2009): "Receptiveness to pluricultural experience reveals the links between different forms of mobility: geographical mobility, of course, bringing a sustained and intense relationship with one or more languages, but also social mobility leading the plurilingual individual to social spaces other than those to which dominant socialisation modes predispose him; also cultural mobility, which may be defined as the ability to update, in life choices, perceptions of 'elsewhere' expressed in latent form in family history" (Coste et al., 2009: 21). The plurilingual individual's strategies consist in keeping a statement of assets (for example, languages as social capital) up to date, and anticipating or controlling their fluctuations (Coste et al., 2009: 21).

Present-day Suriname represents a multilingual reality which is the result of past forms of mobility and is the setting for more recent mobility patterns 
under globalisation that result in what might even be called 'super-diversity' (term from Vertovec 2007). Super-diversity leads to super-diverse linguistic repertoires (Blommaert and Backus 2011, Blommaert and Rampton 2011). Such increasingly complex traces of contact and mobility are evident in the linguistic repertoires of the Surinamese population (for an example of the complex repertoires perceived by Surinamese children, see Léglise and Migge, this volume).

Language attitudes have an important impact on patterns of language use. Language ideologies have been defined as "the cultural system of ideas about social and linguistic relationships, together with their loading of moral and political interests" (Irvine 1989a: 255). "(Language ideologies) are beliefs, or feelings, about languages as used in their social worlds" (Kroskrity 2006: 498). This includes beliefs about the superiority or inferiority of a given language (variety) or beliefs about the (in)appropriateness of a language (variety) in a certain situation or among certain groups of speakers. Language ideologies have to be conceived of as multiple because groups of people tend to be characterised by various degrees of heterogeneity and therefore typically involve different kinds of positionality and produce different kinds of perspectives on the same issue. Language ideologies mediate between social structure and forms of talk, and play an important role in creating and representing social and cultural identities. They are always interested rather than neutral serving the needs and ideas of specific social groups (Kroskrity 2006: 501-510).

Languages too are not bounded entities but are "idealisations that emerge and develop due to socially and historically positioned processes" (Migge and Léglise 2013:112). Language use and language naming practices conceal the fact that languages emerge, change, combine, fade, and do not obediently stay with the social groups with which we (as speakers or researchers) associate them as supreme and obvious markers of ethnic or class identity. The Surinamese case shows that languages cannot be taken for granted as stable entities neatly linked to historical migration and ethnic groups. If ever a simple link would have been possible between a language label and a newly-arrived migrant group, years of assimilation into the state system, hybridisation, and cultural globalisation will have turned such a link into an increasingly complex and changing web of localised meanings, as can be evidenced by the range of different significations of such apparently monolithic terms as Chinees 'Chinese' and Ingi 'Amerindian' in Suriname.

In conclusion, by extending the scope of language contact in Suriname to encompass processes of mobility and identity construction beyond the pale of the urbanised setting, this book offers a new and comprehensive picture 
of language and culture in interaction in present-day Suriname and a situationally nuanced approach to mobility, identity, and language practices and ideologies.

\section{Note}

\section{Onomastics and Spelling Conventions}

Where possible, we use the official Surinamese spelling of place and river names, that is, Donderkamp, Corantijn River, Commewijne River etc. unless quoting from an historical source; Marowijne River is used when mentioned from a Surinamese perspective whereas its French counterpart Maroni is used when referring to the river from a French Guianese perspective.

\section{Languages Spoken in Suriname 2013 and Mentioned in This Book (in Alphabetical Order)}

Amerindian: Kari'na, Lokono, Mawayana, Sikiïyana, Trio, Tunayana-Katwena, Waiwai, Wayana

Asian: Cantonese, Kejia, Putonghua, Sarnámi, Surinamese Javanese

Creole: French Guianese Creole, Haitian Creole, Kwinti, Matawai, Ndyuka, Pamaka, Saamaka, Sranantongo

European: Brazilian Portuguese, Dutch 